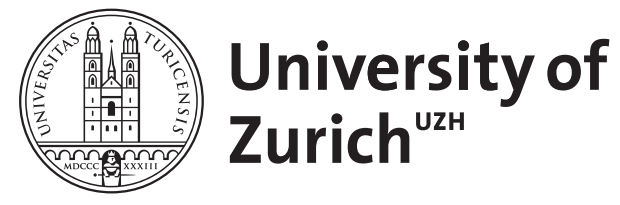

Zurich Open Repository and Archive

University of Zurich

University Library

Strickhofstrasse 39

CH-8057 Zurich

www.zora.uzh.ch

Year: 2013

Ginseng gegen krebsbedingte Müdigkeit - mehr als ein Placebo

Djalali, Sima

DOI: https://doi.org/10.1024/1661-8157/a001498

Posted at the Zurich Open Repository and Archive, University of Zurich

ZORA URL: https://doi.org/10.5167/uzh-90672

Journal Article

Accepted Version

Originally published at:

Djalali, Sima (2013). Ginseng gegen krebsbedingte Müdigkeit - mehr als ein Placebo. Praxis, 102(25):15531554 .

DOI: https://doi.org/10.1024/1661-8157/a001498 


\section{Ginseng gegen krebsbedingte Müdigkeit - mehr als ein Placebo}

\section{Frage:}

Lindert Ginsengextrakt die Beschwerden der krebsbedingten Müdigkeit?

\section{Hintergrund:}

Die krebsbedingte Müdigkeit (KBM) kann zu Einbussen der Leistungsfähigkeit und Lebensqualität führen. Unter Radio- und Chemotherapie tritt dieses Symptom besonders häufig auf, kann jedoch auch nach dem Überlebenden der Krankheit noch jahrelang weiter persistieren. Pharmakologische Psychostimulanzen und Nahrungsergänzungsmittel sind gängige Massnahmen bei KBM, für deren Wirkung es jedoch nur geringe Evidenz gibt. In Studien zur Verträglichkeit, Sicherheit und Dosisfindung (Phase I und II) fanden sich für $2 \mathrm{~g}$ Gingseng pro Tag ermutigende Resultate. Nun liegt eine Phase-III-Studie vor, die den Wirkungsnachweis für Gingseng bei KBM erbringen soll.

\section{Einschlusskriterien:}

- Erwachsene Krebspatienten

- KBM seit mindestens einem Monat, definiert als Müdigkeit von mindestens 4 Punkten auf einer Skala von 0-10

- Aktueller unter oder Status nach Therapie mit kurativer Intention

\section{Ausschlusskriterien:}

- Krebsdiagnose älter als 2 Jahre

- Absehbare Änderungen des Krebstherapieregimes innerhalb der Studienzeit

- Hirntumore, Lymphome des Zentralen Nervensystems

- Schmerzen und Schlaflosigkeit stärker als 4 Punkte auf einer Skala von 0-10

- Einnahme systemischer Steroide oder Opioide

- Einnahme von Gingseng oder anderer Substanzen gegen Fatigue vor Studieneinschluss

\section{Studiendesign und Methode:}

Doppelblinde, randomisierte placebokontrollierte Multizenter-Studie. Daten wurden erhoben bei Baseline, sowie nach 4 und 8 Wochen

\section{Studienort:}

40 Krebstherapiezentren in den USA

\section{Interventionen:}

- Täglich 2 g Gingseng (Panax quinquefolius) für acht Wochen (morgens und abends zwei Kapseln à $500 \mathrm{mg}$ )

\section{Outcome:}

Primärer Outcome

- Änderung der generellen Fatigueausprägung nach 4 und 8 Wochen, gemessen mit dem MFSI-SF-Score (Multidimensional Fatigue Symptom Inventory-Short Form)

Sekundäre Outcomes

- Veränderung physischer, mentaler, emotionaler und antriebsbedingter Fatiguesymptome, gemessen mit dem MFSI-SF-Score

- Veränderung der fatiguebedingten Trägheit und des Antriebs, gemessen mit dem POMSScore (Profile of Mood States) 
- Änderung der Fatigueausprägung nach 4 und 8 Wochen, gemessen mit dem BFI-Score (Brief Fatigue Inventory)

- Nebenwirkungen

\title{
Resultat:
}

- 341 Patienten wurden eingeschlossen, davon beendeten 38 in der Ginseng- und 42 in der Placebogruppe die Studie vorzeitig. Für die Endpunktanalyse standen Daten von 300 Patienten für die Auswertung nach vier Wochen und 272 für die Auswertung nach acht Wochen zur Verfügung. Ginseng- und Placebogruppe unterschieden sich hinsichtlich der Baselinecharakteristika nicht.

- Nach vier Wochen zeigten sich keine statistisch signifikanten Unterschiede zwischen den Gruppen. Nach acht Wochen zeigten sich mit dem MFSI-SF-Score auf zwei (generelle Fatigue und physische Symptome) von fünf untersuchten Subskalen und dem Summenscore statistische Unterschiede zugunsten der Ginsenggruppe, ebenso auf der Subskala des POMS für fatiguebedingte Trägheit. Mit dem BFI-Score waren keine signifikanten Unterschiede zwischen den Gruppen feststellbar.

- 30\% in der Ginsenggruppe vs. 20\% der Kontrollpatienten erlebten eine Verbesserung der Fatiguesymptome um 30\% und mehr. Knapp 10\% vs. 25\% der Patienten erlebten keine Veränderung, bei rund $20 \%$ vs. $30 \%$ verschlimmerten sich die Symptome.

- Als Nebenwirkungen mit einer Häufigkeit von mehr als 1\% traten in beiden Gruppen vergleichbar Agitation, Angstzustände, Schlaflosigkeit, Schwindel und Erbrechen auf.

\section{Kommentar:}

- Die Ergebnisse der Studie weisen darauf hin, dass Ginseng gut verträglich ist und Symptome der krebsbedingten Müdigkeit verbessern kann, sofern über einen längeren Zeitraum angewendet.

- Es ist jedoch zu bedenken, dass Ginseng nicht gleich Ginseng ist und die erhältlichen Extrakte ernte- und weiterverarbeitungsbedingt in ihrem Gehalt an Ginsedoiden - den für die Wirkung verantwortlich gemachten - Substanzen stark schwanken können.

- In der vorliegenden Studie wurden einheitlich Kapseln aus einer Charge mit 3\% Ginsedoidgehalt verwendet. Produkte mit anderer Zusammensetzung könnten andere Effekte aufweisen. Vor allem Extrakte, die mit Methanol gewonnen werden, können östrogene Nebenwirkungen entfalten und Brustkrebszellen zur Proliferation anregen.

- Fazit: Ginsengprodukte, die dem Studienprodukt entsprechen, scheinen Krebspatienten nicht zu schaden, in aktueller Ermangelung an Therapiealternativen für KBM könnte Ginseng eine Option für Patienten mit starkem Leidensdruck sein.

\section{Literatur:}

Barton DL, Liu H, Dakhil SR, et al.: Wisconsin Ginseng (Panax quinquefolius) to Improve Cancer-Related Fatigue: A Randomized, Double-Blind Trial, N07C2. J Natl Cancer Inst 2013; 105(16): 1230-1238.

\author{
Verfasser: \\ Dr. med. Sima Djalali \\ Institut für Hausarztmedizin \\ Universität Zürich \\ Pestalozzistrasse 24 \\ 8091 Zürich
}

